\title{
OPTIMAL DISCRETIZATION OF INVERSE PROBLEMS \\ IN HILBERT SCALES. REGULARIZATION AND SELF-REGULARIZATION OF PROJECTION METHODS*
}

\author{
PETER MATHÉ† AND SERGEI V. PEREVERZEV ${ }^{\ddagger}$
}

\begin{abstract}
We study the efficiency of the approximate solution of ill-posed problems, based on discretized noisy observations, which we assume to be given beforehand. A basic purpose of the paper is the consideration of stochastic noise, but deterministic noise is also briefly discussed. We restrict ourselves to problems which can be formulated in Hilbert scales. Within this framework we shall quantify the degree of ill-posedness, provide general conditions on projection schemes to achieve the best possible order of accuracy. We pay particular attention on the problem of self-regularization vs. Tikhonov regularization.

Moreover, we study the information complexity. Asymptotically, any method which achieves the best possible order of accuracy must use at least such amount of noisy observations.
\end{abstract}

Key words. ill-posed problems, inverse estimation, operator equations, Gaussian white noise, information complexity

AMS subject classifications. $62 \mathrm{G} 05,65 \mathrm{~J} 10$

PII. S003614299936175X

1. Introduction and statement of the main problem. We study optimal discretizations of ill-posed problems in Hilbert scales. On the class of problems, which will be introduced in section 2, the best order of accuracy for a given noise level is well known. But this quantity does not take into account any discretization. Therefore we address two issues.

First, can this best possible order of accuracy be achieved by discretized regularization methods? More precisely, we aim at presenting general conditions, which allow us to achieve this best possible order. This is made explicit for convergence analysis of corresponding schemes of Tikhonov regularization as well as for regularization by projection methods, self-regularization. In particular we pay attention to the limitations of self-regularization and indicate how these naturally occur when the design is given beforehand.

A second issue to be addressed concerns the size, say, $N=N(\delta)$ of the design, necessary for a given noise level $\delta>0$, to enable best order of accuracy. This may be understood as the information complexity of the problem, since, in the asymptotic setting, no numerical method can achieve the best order of accuracy using fewer noisy observations. We establish the asymptotic behavior $N(\delta), \delta \rightarrow 0$.

We shall study ill-posed problems, where we wish to recover some element $x$ from some real Hilbert space $X$ from indirectly observed data near $y=A x$, where $A$ is some injective compact linear operator acting in a real Hilbert space $Y$. For the sake of convenience we will assume that $X=Y$, which can be obtained by isometries. Such linear inverse problems often arise in scientific context, ranging from stereological

${ }^{*}$ Received by the editors September 24, 1999; accepted for publication (in revised form) September 20, 2000; published electronically January 25, 2001. The research of both authors was supported by the Volkswagenstiftung ( $\mathrm{RiP}$-program at Oberwolfach).

http://www.siam.org/journals/sinum/38-6/36175.html

†Weierstraß Institute for Applied Analysis and Stochastics, Mohrenstraße 39, D-10117 Berlin, Germany (mathe@wias-berlin.de).

$\ddagger$ Ukrainian Academy of Sciences, Institute of Mathematics, Tereshenkivska Str. 3, Kiev 4, Ukraine (serg-p@mail.kar.net). 
microscopy (Abel's integral equation) to physical chemistry (Fujita's equation) to satellite geodesy (gravity gradiometry equation).

In practice indirect observations are observed usually in the presence of some noise, so that we observe $y_{\delta}$ given by

$$
y_{\delta}=A x+\delta \xi,
$$

where $\xi$ denotes the normalized noise and $\delta$ is a small positive number used for measuring the noise level.

Suppose further that even the observations (1.1) cannot be observed exactly, but they can only be observed in discretized or binned form. To be more precise, we have only a vector $\varphi\left(y_{\delta}\right)=\left\{y_{\delta, i}\right\}_{i=1}^{n} \in \mathbb{R}^{n}$ defined by

$$
y_{\delta, i}=\left\langle y_{\delta}, \varphi_{i}\right\rangle=\left\langle A x, \varphi_{i}\right\rangle+\delta\left\langle\xi, \varphi_{i}\right\rangle, \quad i=1, \ldots, n,
$$

where $\langle$,$\rangle denotes the inner product in Y, \varphi_{i}, i=1, \ldots, n$ is some orthonormal system, usually called design.

When fixing a design $\left\{\varphi_{1}, \ldots, \varphi_{n}\right\}$ we may rewrite (1.2) as

$$
Q_{n}\left(y_{\delta}\right)=Q_{n}(A x+\delta \xi),
$$

where $Q_{n}$ denotes the orthogonal projection onto $\operatorname{span}\left\{\varphi_{1}, \ldots, \varphi_{n}\right\}$.

At this point it is important to note that we assume to have observations without repetitions, which means that each of the functionals $\left\langle y_{\delta}, \varphi_{i}\right\rangle$ is observed only once. The problem becomes different if we allow repetitions. In that case the behavior depends very much on the kind of noise.

For deterministic noise we do not gain from repetitions; only the space spanned by the design elements is important. If we know that the noise is random and independent, then we may use this to decrease the noise level at each functional by suitable repetitions. Under these circumstances one can asymptotically even achieve arbitrary accuracy.

From our discussion above we can summarize that given (1.1) and assuming that the unknown solution belongs to some set $\mathcal{M}$ and the noise is either deterministic or random, the ill-posed problem is completely characterized by the triple $(A: X \rightarrow Y, \mathcal{M}, \delta)$, where $Y$ indicates the space where noisy observations are given, $X$ denotes the space in which we agree to measure the accuracy. The operator $A$ in (1.1) determines the way the observations are indirect. The set $\mathcal{M} \subset X$ describes our a priori knowledge on the exact solution and $\delta>0$ indicates the noise level. We shall think of $(A: X \rightarrow Y, \mathcal{M}, \delta)$ as being the mathematical problem under consideration.

This mathematical problem is accompanied with a numerical one. When seeking approximate solutions to a given mathematical problem $(A: X \rightarrow Y, \mathcal{M}, \delta)$, we have to specify the class of admissible numerical methods. This class will generically be denoted by $\mathcal{U}$. Each method $u \in \mathcal{U}$ must be based on some design, say, $\left\{\varphi_{1}, \ldots, \varphi_{n}\right\}$, which describes the way we obtain noisy observations; see (1.2), (1.3). The resulting approximation based on such design may be obtained by any (measurable) mapping $S: \mathbb{R}^{n} \rightarrow X$, hence

$$
u\left(y_{\delta}\right)=S\left(\left\langle y_{\delta}, \varphi_{1}\right\rangle,\left\langle y_{\delta}, \varphi_{2}\right\rangle, \ldots,\left\langle y_{\delta}, \varphi_{n}\right\rangle\right) .
$$

Let $\mathcal{U}_{n}$ denote the class of all methods based on design of at most $n$ elements. We assume that $\mathcal{U}=\bigcup_{n=1}^{\infty} \mathcal{U}_{n}$ and find it convenient to denote $(A: X \rightarrow Y, \mathcal{U}, \mathcal{M}, \delta)$, the numerical problem, corresponding to the mathematical problem $(A: X \rightarrow Y, \mathcal{M}, \delta)$. 
There is a considerable literature concerned with deterministic noise, the classical approach to inverse problems. We mention Tikhonov and Arsenin [37], Morozov [23], Vaĭnikko and Veretennikov [40], Traub, Wasilkowski, and Woźniakowski [38], Louis [18], Werschulz [42], and Engl, Hanke, and Neubauer [11]. On the other hand, for stochastic noise, in which case we deal with a statistical problem, we refer to Wahba [41], Nychka and Cox [29], Johnstone and Silverman [15], Nussbaum [27], Donoho [9], Mair and Ruymgaart [21], Golubev and Khasminskii [13], Lukas [19], Cavalier and Tsybakov [5], and Chow, Ibragimov, and Khasminskii [6].

As far as the design is concerned it is sometimes possible to choose it. In this case one might prefer to choose elements from the singular value decomposition of the operator $A$ or the wavelet-vaguelette decomposition. For such designs the recovery of $x$ from noisy data as in (1.2) was studied by Schock [34] and more recently by Johnstone and Silverman [15], Donoho [9], and Golubev and Khasminskii [13]. Often the design is given and independent of the operator. An example for this is the estimation of a probability density function $x$ from discretized (binned) data or histogram as $y_{\delta}$. In this case we assume that the operator $A$ is of the form

$$
A x(t)=\int_{0}^{1} a(t, \tau) x(\tau) d \tau
$$

acting in $X=Y=L_{2}(0,1)$. A particular example for this is Abel's equation; see Example 2 below. The data are given as averages of histogram bins $\left[u_{i-1, n}, u_{i, n}\right)$ with bin limits $0=u_{0, n}<u_{1, n}<\cdots<u_{n, n}=1$, i.e.,

$$
y_{\delta, i}(t)=\frac{1}{u_{i, n}-u_{i-1, n}} \int_{u_{i-1, n}}^{u_{i, n}} A x(u) d u+\varepsilon_{i}, \quad t \in\left[u_{i-1, n}, u_{i, n}\right), \quad i=1, \ldots, n
$$

This corresponds to (1.3) with design $\varphi_{i}=\varphi_{i}(t)=\left(u_{i, n}-u_{i-1, n}\right)^{-1 / 2} \chi_{i, n}(t), \varepsilon_{i}=$ $\delta\left\langle\varphi_{i}, \xi\right\rangle \varphi_{i}$, where $\chi_{i, n}$ is the characteristic function of the interval $\left[u_{i-1, n}, u_{i, n}\right)$. The approximate solution of Abel's integral equation based on histograms was studied by Nychka and Cox [29]. It is easy to verify that such histogram design corresponds to neither the singular value decomposition nor the wavelet-vaguelette decomposition of the Abel integral operator, for example.

It is the aim of this paper to study efficiency issues for recovering the unknown element $x$ from indirect and noisy discrete observations, as described above in terms of the numerical problem $(A, \mathcal{U}, \mathcal{M}, \delta)$.

For any given method $u \in \mathcal{U}$, its error at the exact solution $x$ will be measured as

$$
e^{\operatorname{det}}(A, u, x, \delta)=\sup \left\{\left\|x-u\left(y_{\delta}\right)\right\|_{X}, \quad\|\xi\|_{Y} \leq 1\right\}
$$

for the case of deterministic noise. For the stochastic case the white noise model (see, for example, Chow, Ibragimov, and Khasminskii [6], Donoho [9], and Cavalier and Tsybakov [5]) is widely accepted. Here $\xi$ is a weak or generalized random element such that for any $f \in Y$ the inner product $\langle\xi, f\rangle$ is a zero mean Gaussian random variable on a probability space $(\Omega, \Sigma, \mathbb{P})$ with variance $\|f\|^{2}$. In addition, for any $f, g \in Y$

$$
\mathbf{E}\langle f, \xi\rangle\langle g, \xi\rangle=\langle f, g\rangle,
$$

where $\mathbf{E}$ is the expectation with respect to $\mathbb{P}$. At this point we would like to note that the normal distribution of the random noise will be important only for the proof of 
optimal lower bounds. To obtain the upper bounds for the accuracy of regularization methods under study we in fact do not impose any restrictions on the probability distribution of $\xi$. It will be sufficient to assume that $\xi$ is some zero mean stochastic process with bounded covariance operator.

Within the framework of white noise the error of any given method $u \in \mathcal{U}$ is measured by the risk

$$
e^{r a n}(A, u, x, \delta)=\left(\mathbf{E}\left\|x-u\left(y_{\delta}\right)\right\|_{X}^{2}\right)^{1 / 2} .
$$

As usual, the uniform error over $x \in \mathcal{M}$ is defined to be the supremum over pointwise errors with respect to $x \in \mathcal{M}$, e.g., for stochastic noise we let $e^{r a n}(A, u, \mathcal{M}, \delta)=$ $\sup _{x \in \mathcal{M}} e^{r a n}(A, u, x, \delta)$. These quantities measure the quality of some specific method $u$. If we let $u$ vary within class $\mathcal{U}$ of methods, then we may consider the minimal error within this class, $e^{r a n}(A, \mathcal{U}, \mathcal{M}, \delta)=\inf _{u \in \mathcal{U}} e^{r a n}(A, u, \mathcal{M}, \delta)$, and the corresponding version $e^{\operatorname{det}}(A, \mathcal{U}, \mathcal{M}, \delta)$ for deterministic noise. In accordance with usual terminology in information-based complexity (see [38]), we denote

$$
r_{n}^{\text {ran }}(A, \mathcal{M}, \delta):=e^{r a n}\left(A, \mathcal{U}_{n}, \mathcal{M}, \delta\right)
$$

the $n$th minimal radius of information within class $\mathcal{U}$ and $r_{n}^{\text {det }}(A, \mathcal{M}, \delta)$ its deterministic counterpart.

A lower bound for $e^{r a n}(A, \mathcal{U}, \mathcal{M}, \delta)$ is certainly given by

$$
\mathcal{E}^{r a n}(A, \mathcal{M}, \delta):=\inf _{u: Y \rightarrow X} \sup _{x \in \mathcal{M}}\left(\mathbf{E}\|x-u(A x+\delta \xi)\|_{X}^{2}\right)^{1 / 2}
$$

with the corresponding version for deterministic noise. Above, the inf is taken over all possible (measurable) mappings. Most investigations on ill-posed problems have centered around quantities of such a type.

Within the classical framework of optimal recovery (formally we may let $\delta=0$ ) we have $r_{n}^{r a n}(A, \mathcal{M}, 0)=r_{n}^{\operatorname{det}}(A, \mathcal{M}, 0) \rightarrow 0$, as $n$ increases, which means that we can recover $x$ with arbitrary accuracy by enlarging the design properly. In contrast, under the presence of noise $\delta>0$ the sequence $r_{n}^{r a n}(A, \mathcal{M}, \delta)$ will decrease, but there will be a positive limit (see, e.g., Corollary 1)

$$
\lim _{n \rightarrow \infty} r_{n}^{r a n}(A, \mathcal{M}, \delta)=e^{r a n}(X, \mathcal{U}, \mathcal{M}, \delta) \geq \mathcal{E}^{r a n}(A, \mathcal{M}, \delta)>0,
$$

depending on the noise level. This limit cannot be beaten by any numerical method within class $\mathcal{U}$.

Thus we measure the quality of any numerical method $u$ against this lower bound. For this purpose we fix $C>1$ and seek for $n$ satisfying $r_{n}^{\operatorname{ran}}(A, \mathcal{M}, \delta) \leq$ $C e^{r a n}(A, \mathcal{U}, \mathcal{M}, \delta)$. This results in a number $n=n(\delta)$ which measures the minimal amount of information necessary to recover $x$ by methods from class $\mathcal{U}$ with best possible accuracy up to constant $C$,

$$
N^{r a n}(A, \mathcal{U}, \mathcal{M}, \delta):=\inf \left\{n, \quad r_{n}^{r a n}(A, \mathcal{M}, \delta) \leq C e^{r a n}(A, \mathcal{U}, \mathcal{M}, \delta)\right\}
$$

and the respective version within the framework of deterministic noise. We agree to call these numbers the information complexity of the numerical problem $(A, \mathcal{U}, \mathcal{M}, \delta)$ under random or deterministic noise, respectively.

Thus our focus will be on two problems. Given a numerical problem, can we describe the asymptotic behavior of the information complexity? We shall establish 
lower bounds and then indicate how these bounds can be achieved by regularization methods based on projection schemes. Moreover, we will discuss whether selfregularization of projection methods based on given design is possible or if some other regularization is required.

2. Degree of ill-posedness in Hilbert scales. Assume that for the mathematical problem $(A: X \rightarrow Y, \mathcal{M}, \delta)$, where $\delta$ describes the level of noise in the data, one wishes to ensure that any possible solution $x_{\delta}$ also varies within a ball, proportional to $\delta$. It is easy to see that this is exactly the case if $\left\|A^{-1}: Y \rightarrow X\right\|<\infty$. Otherwise the problem is ill-posed and we lose accuracy when recovering $x$ from noisy data. Thus we want to assign each mathematical problem its degree of ill-posedness, a notion which was first coined by Wahba [41]. If we formulate the problem in parametric scales of spaces, then we may express the degree in terms of these parameters. Therefore we shall study problems in Hilbert scales.

A Hilbert scale $\left\{X^{\lambda}\right\}_{\lambda \in \mathbb{R}}$ is a family of Hilbert spaces $X^{\lambda}$ with inner product $\langle u, v\rangle_{\lambda}:=\left\langle L^{\lambda} u, L^{\lambda} v\right\rangle$, where $L$ is a given unbounded strictly positive self-adjoint operator in a dense domain of some initial Hilbert space, say $X$. To be more precise, $X^{\lambda}$ is defined as the completion of the intersection of domains of the operators $L^{\nu}, \nu \geq$ 0 , accomplished with norm $\|\cdot\|_{\lambda}$ defined as $\|x\|_{\lambda}:=\langle x, x\rangle_{\lambda}^{1 / 2}=\left\|L^{\lambda} x\right\|_{0}$, where $\|\cdot\|_{0}=\|\cdot\|$ is the norm in $X$.

Hilbert scales $\left\{X^{\lambda}\right\}$ are invariant with respect to rescaling $\lambda \rightarrow a \lambda+b$, for $a>0, b \in \mathbb{R}$. Since $\left\{X^{\lambda}\right\}$ are usually specific Sobolev spaces, say, $H^{\lambda}(0,1)$ and for definiteness of scaling we assume $\lambda$ be chosen to fit the usual smoothness as, e.g., in $H^{\lambda}(0,1)$. This goal is achieved by assuming that the canonical embeddings $J_{\lambda}: X^{\lambda} \rightarrow X, \lambda>0$ obey

$$
a_{n}\left(J_{\lambda}\right) \asymp n^{-\lambda}
$$

where $\asymp$ means equivalent in order, and $a_{n}$ denotes the $n$th approximation number (see [30]); for example,

$$
a_{n}\left(J_{\lambda}\right):=\inf \left\{\left\|J_{\lambda}-U\right\|_{X^{\lambda} \rightarrow X}, \quad \operatorname{rank} U<n\right\} .
$$

By studying mathematical problems $(A: X \rightarrow Y, \mathcal{M}, \delta)$, for an operator $A$, which initially acts within $X$, in Hilbert scales we mean that both the domain $X$ and the target $Y$ belong to appropriate Hilbert scales, $X \in\left\{X^{\lambda}\right\}, Y \in\left\{Y^{\lambda}\right\}$, which are linked by assuming $X^{0}=Y^{0}=X$ (actually only a finite segment of parameters $\lambda \in \mathbb{R}$ will be involved). Moreover, we assume that the scaling for $\left\{Y^{\lambda}\right\}$ is the same as for $\left\{X^{\lambda}\right\}$. When indicating norms in spaces $X^{\lambda}$ or $Y^{\lambda}$, we shall suppress the symbol for the space and just mention the parameter. It will be transparent from the context which scale is meant.

The basic assumption concerning the operator $A$ is as follows: For some parameter $a>0$ and for any $\lambda \in \mathbb{R}$ the range of $A: X^{\lambda-a} \rightarrow Y^{\lambda}$ coincides with $Y^{\lambda}$ and there exist constants $D, d>0$ such that

$$
d\|x\|_{\lambda-a} \leq\|A x\|_{\lambda} \leq D\|x\|_{\lambda-a}
$$

for all $x \in X^{\lambda-a}$. In other words, the operator $A$ acts along the Hilbert scales with step $a$ as isomorphism between pairs $X^{\lambda-a}$ and $Y^{\lambda}$.

There is substantial literature devoted to inverse problems with operators acting along Hilbert scales. We mention Natterer [25], Neubauer [26], Mair [20], Hegland [14], 
Tautenhahn [36], and Dicken and Maass [8]. These papers studied only problems with deterministic noise. Moreover, the recovery problem from finitely many observations, i.e., a finite design with elements not necessarily depending on the operator $A$, was not considered.

We illustrate this setup by two examples.

Example 1. We introduce Symm's equation

$$
\int_{\Gamma} \log (|u-v|) z(v) d S_{v}=g(u), \quad u \in \Gamma,
$$

arising from the Dirichlet boundary value problem for the Laplace equation in a region with boundary curve $\Gamma$. We assume that $\Gamma$ admits a $C^{\infty}$-smooth 1 -periodic parametrization $\gamma:[0,1] \rightarrow \Gamma$ and the logarithmic capacity cap $\Gamma \neq 1$. Then we can rewrite (2.3) as

$$
A x(t):=\int_{0}^{1} \log (|\gamma(t)-\gamma(\tau)|) x(\tau) d \tau=y(t), \quad t \in[0,1]
$$

where $x(t):=z(\gamma(t))\left|\gamma^{\prime}(t)\right|$ and $y(t):=g(\gamma(t))$. It can be seen that the operator $A$ obeys condition (2.2) with $a=1$ within the scale $X^{\lambda}=Y^{\lambda}:=H^{\lambda}(0,1), \lambda \in \mathbb{R}$, of Sobolev spaces of 1-periodic functions (distributions); see, e.g., Bruckner, Prössdorf, and Vainikko [4] for details. Further examples of integral operators can be found in Neubauer [26] and Mair and Ruymgaart [21].

Often a given operator does not fit any of the known scales. However, in many cases one can construct the scales adapted to the operator. This is the case when $A$ acts compactly and injectively in some Hilbert space $X$. Then $A$ meets condition (2.2) with $a=1 / 2$ in the scales $\left\{X^{\lambda}\right\}$ and $\left\{Y^{\lambda}\right\}$, generated by the operators $L:=\left(A^{*} A\right)^{-1}, L_{1}:=\left(A A^{*}\right)^{-1}$, respectively; see Natterer [25] and Hegland [14]. Indeed, by definition

$$
\|A u\|_{\lambda}=\left\|\left(A A^{*}\right)^{-\lambda} A u\right\|=\left\|A\left(A^{*} A\right)^{-\lambda} u\right\|=\left\|\left(A^{*} A\right)^{\frac{1}{2}-\lambda} u\right\|=\|u\|_{\lambda-\frac{1}{2}} .
$$

It should, however, be noted that further verification is required to see that scaling is according to (2.1). We illustrate this by introducing the following important example.

Example 2. Let $X=L_{2}(0,1)$ and the operator $A$ be Abel's integral operator

$$
A x(t):=\frac{1}{\sqrt{\pi}} \int_{t}^{1} \frac{x(\tau)}{\sqrt{\tau-t}} d \tau, \quad t \in(0,1) .
$$

If now $\left\{X^{\lambda}\right\},\left\{Y^{\lambda}\right\}$ are generated by $L:=\left(A^{*} A\right)^{-1}, L_{1}:=\left(A A^{*}\right)^{-1}$, where

$$
A^{*} x(t)=\frac{1}{\sqrt{\pi}} \int_{0}^{t} \frac{x(\tau)}{\sqrt{t-\tau}} d \tau,
$$

then it follows from the result by Dostanić [10] that $a_{n}\left(A^{*}: X \rightarrow X\right) \asymp n^{-1 / 2}$ and we indeed have

$$
a_{n}\left(J_{\lambda}: X^{\lambda} \rightarrow X\right)=a_{n}\left(\left(A^{*} A\right)^{\lambda}\right)=a_{n}\left(A^{*}\right)^{2 \lambda} \asymp n^{-\lambda}, \quad \lambda>0 .
$$

Returning to our problem $(A, \mathcal{M}, \delta)$ we assume that the noisy data are given in $Y=Y^{0}=X$ and we measure accuracy in $X^{\nu}$. Suppose now that $A: X^{\nu} \rightarrow X$ has a bounded inverse. Then we may formally invert it and rewrite (1.1) as

$$
x=A^{-1} y_{\delta}-\delta A^{-1} \xi .
$$


Therefore, if the noise is deterministic with $\|\xi\|_{X} \leq 1$, and $A$ has a bounded inverse, then in principle we can recover the unknown solution $x$ up to order $\delta$; otherwise it might be ill-posed and the quantity

$$
\alpha:=\sup \left\{\lambda, \quad\left\|A^{-1}: X \rightarrow X^{\lambda}\right\|<\infty\right\}
$$

is less than or equal to $\nu$. Therefore it is natural to define $(\nu-\alpha)_{+}$to be the degree of ill-posedness.

In case the operator $A$ obeys property (2.2) for some $a$, the supremum in condition $(2.7)$ is attained and $(\nu-\alpha)_{+}=(\nu+a)_{+}$is the degree of ill-posedness of the operator $A$. We mention that for $\nu=0$ the notion of degree of ill-posedness coincides with the one introduced by Wahba [41].

For stochastic noise the situation is a little more difficult. Even if we arrive at (2.6), and assume that the operator $A$ has a bounded inverse, then, since the noise is stochastic, we cannot guarantee that the accuracy is kept, since $\mathbf{E}\left\|A^{-1} \xi\right\|_{\nu}^{2}$ may be unbounded. Therefore, analogous to the deterministic setting, we will say that the problem is well-posed if the above expectation is bounded and ill-posed otherwise. If the latter is the case, then we let

$$
\alpha:=\sup \left\{\lambda, \quad \mathbf{E}\left\|A^{-1} \xi\right\|_{\lambda}^{2}<\infty\right\} .
$$

Again we agree do denote the degree of ill-posedness by $(\nu-\alpha)_{+}$, now for white noise.

For such noise we can compute the degree of ill-posedness. We shall sketch the proof under the additional assumption that the operator $A$ and the generator $L$ of the Hilbert scale $\left\{X^{\lambda}\right\}$ are properly related. Following Mair and Ruymgaart [21] we assume that the eigenvectors of the operator $L$ coincide with the eigenvectors of $A^{*} A$. This means that both the operator $L^{-1}$ and the operator $A$ can be represented in the form

$$
L^{-1} g=\sum_{k=1}^{\infty} l_{k}\left\langle g, f_{k}\right\rangle f_{k}, \quad A g=\sum_{k=1}^{\infty} \gamma_{k}\left\langle g, f_{k}\right\rangle u_{k}
$$

where $\left\{f_{k}\right\},\left\{u_{k}\right\}$ are some orthonormal bases of $X$. Taking into account assumptions (2.1), (2.2), and (2.8), we see that necessarily

$$
l_{k} \asymp k^{-1}, \quad \gamma_{k} \asymp k^{-a} .
$$

We have the following proposition.

Proposition 2.1. An operator A which obeys assumptions (2.8) and (2.9) has degree of ill-posedness $(\nu+a+1 / 2)_{+}$when the noise in (1.1) is assumed to be white noise.

Remark 1. Actually this result can be proven, replacing assumptions (2.8) and (2.9) by (2.1), (2.2). Then the proof is more involved and uses $s$-number arguments.

Proof. Suppose we have fixed some $\lambda$ for which $\mathbf{E}\left\|A^{-1} \xi\right\|_{\lambda}^{2}<\infty$. Then from (2.8) it follows that

$$
L^{\lambda} A^{-1} \xi=\sum_{k=1}^{\infty} l_{k}^{-\lambda} \gamma_{k}^{-1}\left\langle u_{k}, \xi\right\rangle f_{k}
$$

Using (2.9) and (1.6) we arrive at

$$
\mathbf{E}\left\|A^{-1} \xi\right\|_{\lambda}^{2}=\mathbf{E}\left\|L^{\lambda} A^{-1} \xi\right\|^{2}=\sum_{k=1}^{\infty} l_{k}^{-2 \lambda} \gamma_{k}^{-2} \asymp \sum_{k=1}^{\infty} k^{2(\lambda+a)}<\infty,
$$


which is true whenever $\lambda<-a-1 / 2$. Thus

$$
\alpha:=\sup \left\{\lambda, \mathbf{E}\left\|A^{-1} \xi\right\|_{\lambda}^{2}<\infty\right\}=\sup \{\lambda, \lambda<-a-1 / 2\}=-a-1 / 2,
$$

which corresponds to a degree of ill-posedness of $(\nu+a+1 / 2)_{+}$.

We end our short digression with the remark that for deterministic noise the bound $\alpha$ was attained, assuming property (2.2). For white noise this is not the case; but as we will see below, the best possible accuracy will nevertheless be reflected by the degree of ill-posedness as just defined.

In addition, we assumed that the actual solution belongs to $\mathcal{M} \subset X^{\nu}$. If $\mathcal{M}$ is a ball in some $X^{\mu}$, then this requires $\mu \geq \nu$ and we let $\mu-\nu$ be the effective smoothness of $\mathcal{M}$. Thus our problem is characterized by $(\nu+a)_{+}$and $(\mu-\nu)$ in the deterministic case, whereas for white noise these parameters are $(\nu+a+1 / 2)_{+}$and $(\mu-\nu)$.

3. Minimal errors: Lower bounds. In this section we shall provide lower bounds for minimal errors for the numerical problem $(A, \mathcal{U}, \mathcal{M}, \delta)$, using the asymptotics of the corresponding best possible accuracies $\mathcal{E}^{\text {det }}(A, \mathcal{M}, \delta)$ and $\mathcal{E}^{\text {ran }}(A, \mathcal{M}, \delta)$. For this purpose we fix $\mathcal{M}$ being the ball $X_{R}^{\mu}$ in $X^{\mu}$ of radius $R>0$.

We first note that in the deterministic setting, as in our discussion above, we have well-posedness for $\nu \leq-a$. This yields $\mathcal{E}^{\text {det }}(A, \mathcal{M}, \delta) \asymp \delta$. For $\nu>-a$, the asymptotics of $\mathcal{E}^{\operatorname{det}}(A, \mathcal{M}, \delta)$ is well known (see, e.g., Natterer [25] and Tautenhahn [36], but also [11]):

$$
\mathcal{E}^{\operatorname{det}}\left(A, X_{R}^{\mu}, \delta\right) \asymp \delta^{\frac{\mu-\nu}{(\mu-\nu)+(\nu+a)+}} .
$$

Next we shall obtain the stochastic analogue of estimate (3.1).

Proposition 3.1. Let the assumptions (2.1), (2.2), and (2.8) be fulfilled. Then for $\mu, a>0$ and $\nu \in[-a, \mu]$ we have

$$
\mathcal{E}^{r a n}\left(A, X_{R}^{\mu}, \delta\right) \asymp \delta^{\frac{\mu-\nu}{(\mu-\nu)+(\nu+a+1 / 2)}} .
$$

The proof relies on the following lemma, originally proven in Korostelev and Tsybakov [16, Chapt. 9]; see also [2].

LEMMA 3.2. Suppose we are given

$$
v_{k}=\theta_{k}+\delta \sigma_{k} \xi_{k}, \quad k=1,2, \ldots,
$$

where $\xi_{k}$ are independently and identically distributed (i.i.d) $N(0,1), \sigma_{k} \asymp k^{b}$ and $\theta=\left(\theta_{1}, \theta_{2}, \ldots\right)$ is unknown but belongs to

$$
B_{R}^{s}:=\left\{\theta: \sum_{k} \lambda_{k}^{2} \theta_{k}^{2} \leq R^{2}, \lambda_{k} \asymp k^{s}\right\} .
$$

Then for $b, s>0$ the following asymptotics holds:

$$
\inf _{\hat{\theta}(v)} \sup _{\theta \in B_{R}^{s}} \mathbf{E}\|\theta-\hat{\theta}(v)\|_{l_{2}}^{2} \asymp \delta^{\frac{2 s}{s+b+1 / 2}},
$$

where the inf is taken over all estimators $\hat{\theta}(v)$ based on observations (3.2).

Proof of Proposition 3.1. Using (2.8) and (2.9) we can represent the observations (1.1) in the equivalent form (3.2) with $v_{k}=\gamma_{k}^{-1} l_{k}^{-\nu}\left\langle y_{\delta}, u_{k}\right\rangle, \sigma_{k}=\gamma_{k}^{-1} l_{k}^{-\nu} \asymp k^{\nu+a}$, 
$\xi_{k}=\left\langle\xi, u_{k}\right\rangle$ and $\theta_{k}=\left\langle L^{\nu} x, f_{k}\right\rangle, \quad k=1,2, \ldots$ Here it is important that no repetitions are allowed. Moreover, it follows from our assumptions (see (1.6)) that $\xi_{k}=\left\langle\xi, u_{k}\right\rangle$ are Gaussian pairwise uncorrelated random variables and as a consequence they are i.i.d. $N(0,1)$. Since we assumed $x \in X_{R}^{\mu}$, the ball in $X^{\mu}$ of radius $R$, we conclude that $\theta:=\left(\theta_{1}, \theta_{2}, \ldots\right) \in B_{R}^{s}$ for $s:=\mu-\nu$ and $\lambda_{k}=l_{k}^{-(\mu-\nu)}$. Indeed,

$$
\sum_{k=1}^{\infty} \lambda_{k}^{2} \theta_{k}^{2}=\sum_{k=1}^{\infty} l_{k}^{-2(\mu-\nu)}\left\langle L^{\nu} x, f_{k}\right\rangle^{2}=\sum_{k=1}^{\infty} l_{k}^{-2 \mu}\left\langle x, f_{k}\right\rangle^{2}=\left\|L^{\mu} x\right\|^{2} \leq R^{2} .
$$

Note that any estimator $\hat{\theta}(v)$ of $\theta$ based on observations (3.2) yields an approximation $u:=\sum_{k=1}^{\infty} \hat{\theta}_{k}(v) f_{k}$ for $L^{\nu} x$ and vice versa. Therefore, applying Lemma 3.2 with $b:=\nu+a$ and $s:=\mu-\nu$ we obtain the desired asymptotics

$$
\mathcal{E}^{r a n}\left(A, X_{R}^{\mu}, \delta\right) \asymp \delta^{\frac{\mu-\nu}{\mu+a+1 / 2}},
$$

completing the proof.

Comparing the asymptotics from Proposition 3.1 with the one in (3.1) we conclude that indeed white noise introduces an additional degree $1 / 2$ of ill-posedness, this time caused by the stochastic nature. This influence of white noise to the degree of illposedness was observed by Nussbaum [27] for the special case when the operator $A$ denotes $a$-fold integration considered in Sobolev spaces. He also gave a heuristic explanation; see [28].

We turn to the main result of this section, a lower bound for the $n$th minimal radius of information within the class $\mathcal{U}$ of methods based on designs consisting of linear functionals, as in (1.2). Recall that given $n$ we denote by $\mathcal{U}_{n}$ the class of all methods based on design of at most $n$ elements.

Corollary 1. Under the assumptions of Proposition 3.1 we have for $\nu \in[-a, \mu]$ the lower bound

$$
r_{n}^{r a n}\left(A, X_{R}^{\mu}, \delta\right) \geq c\left\{n^{-(\mu-\nu)}+\delta^{\frac{\mu-\nu}{\mu+a+1 / 2}}\right\} .
$$

Proof. To make arguments easier, we consider $x$ as well as $u\left(y_{\delta}\right)$ as elements in $L_{2}\left(X^{\nu}\right)$; thus we rewrite $(1.7)$ as

$$
e^{r a n}(A, u, x, \delta)=\left(\mathbf{E}\left\|x-u\left(y_{\delta}\right)\right\|_{\nu}^{2}\right)^{1 / 2}=\left\|x-u\left(y_{\delta}\right)\right\|_{L_{2}\left(X^{\nu}\right)} .
$$

To shorten notation we assume that each method $u \in \mathcal{U}_{n}$ uses its own design $\varphi_{1}, \ldots, \varphi_{n}$. We now estimate

$$
\begin{aligned}
& r_{n}^{r a n}\left(A, X_{R}^{\mu}, \delta\right) \geq \inf _{u \in \mathcal{U}_{n}} \sup _{\substack{x \in X_{R}^{\mu} \\
\left\langle A x, \varphi_{i}\right\rangle=0 \\
i=1, \ldots, n}}\|x-u(\delta \xi)\|_{L_{2}\left(X^{\nu}\right)} \\
& \geq \inf _{u \in \mathcal{U}_{n}} \sup _{\substack{x \in X_{R}^{\mu} \\
\left\langle A x, \varphi_{i}\right\rangle=0 \\
i=1, \ldots, n}} \max \left\{\|x-u(\delta \xi)\|_{L_{2}\left(X^{\nu}\right)},\|x+u(\delta \xi)\|_{L_{2}\left(X^{\nu}\right)}\right\} \\
& \geq \inf _{u \in \mathcal{U}_{n}} \sup _{\substack{x \in X_{R}^{\mu} \\
\left\langle A x, \varphi_{i}\right\rangle=0 \\
i=1, \ldots, n}}\|x\|_{L_{2}\left(X^{\nu}\right)} \\
& =\inf _{u \in \mathcal{U}_{n}} \sup _{\substack{x \in X_{R}^{\mu} \\
\left\langle A x, \varphi_{i}\right\rangle=0 \\
i=1, \ldots, n}}\|x\|_{\nu} \geq \inf _{\varphi_{1}, \ldots, \varphi_{n} \in X^{-\mu}} \sup _{\substack{x \in X_{R}^{\mu} \\
\left\langle x, \varphi_{i}\right\rangle=0 \\
i=1, \ldots, n}}\|x\|_{\nu} .
\end{aligned}
$$


The last expression in (3.3) is just $R$ times the $(n+1)$ st Gelfand number of the embedding $J_{\mu}^{\nu}: X^{\mu} \rightarrow X^{\nu}$; see [22] for details on optimal approximative methods and $s$-numbers. Since for operators acting between Hilbert spaces Gelfand and approximation numbers coincide (see ibid.), it follows from (2.1) that

$$
r_{n}^{r a n}\left(A, X_{R}^{\mu}, \delta\right) \geq R a_{n}\left(J_{\mu}^{\nu}\right) \asymp n^{-(\mu-\nu)} .
$$

Since, on the other hand, by the very definition

$$
r_{n}^{r a n}\left(A, X_{R}^{\mu}, \delta\right) \geq \mathcal{E}^{r a n}\left(A, X_{R}^{\mu}, \delta\right) \asymp \delta^{\frac{\mu-\nu}{\mu+a+1 / 2}},
$$

we arrive together with estimate (3.4) at the statement of Corollary 1.

4. Discretized noisy observations as data for projection schemes. Regularization and self-regularization of projection methods. Note that (1.3) is the standard form of a projection scheme for the approximate solution of the noisy operator equation (1.1). However, if this is ill-posed, then some regularization may be required for solving (1.3).

The classical approach to regularization was proposed by Tikhonov; its application to nondiscretized equations (1.1) in Hilbert scales was analyzed by Natterer [25].

Another approach to the approximate solution of (1.1) was undertaken by Engl and Neubauer [12] and Neubauer [26], who studied semidiscrete methods. Within the framework of semidiscrete methods the operator equation is discretized only from one side, passing from (1.1) to

$$
A P_{m, s} x=y_{\delta},
$$

where $P_{m, s}$ denotes the orthogonal projection (now in some $X^{s}$ ) onto some finite dimensional subspace $V_{m} \subset X^{s}$. If we apply such a scheme, then this will result in a discretization of the data $y_{\delta}$ with respect to a basis which is determined by the operator $A$, as will be discussed in Remark 2 in section 5. However, as the example of binned data (1.5) shows, the discretization with respect to the noisy data $y_{\delta}$ is often given beforehand.

For this reason it is important to study the "two-sided" discretization of (1.1), namely,

$$
Q_{n} A P_{m, s} x=Q_{n} y_{\delta}
$$

The discrete equation (4.2) may not require further regularization by Tikhonov's method. Instead it may happen that regularization may be achieved by just choosing the discretization parameters properly. This is called self-regularization; see Natterer [24], which is based on a stability property; see Lemma 6.2 below. To the best of our knowledge, all projection schemes which were applied and enjoy the property of self-regularization may in principle be derived from the standard form of the least-square method, as introduced in (6.1). They necessarily lead to a one-sided discretization as in (4.1), and hence require specific projections $Q_{n}$.

Therefore we will study a modification of least-square methods for two-sided discretization. We add that in all previous study of self-regularization only deterministic noise was considered.

We close this section mentioning that assumptions to be made on the design which enable self-regularization are more restrictive than for Tikhonov regularization. Therefore, if these assumptions are not met, then some different regularization is unavoidable. We shall provide an example in section 7 . 
5. Tikhonov regularization of projection schemes in Hilbert scales. The classical variant of Tikhonov regularization based on observations (1.2) or in equivalent form (1.3) is obtained by minimizing the functional

$$
\left\|Q_{n} A x-Q_{n} y_{\delta}\right\|^{2}+\alpha\|x\|_{s}^{2}
$$

over some finite dimensional subspace $V_{m} \subset X^{s}$, where we assume that the true solution $x_{0}=A^{-1} y \in X_{R}^{\mu} \subset X^{s}$. In statistics this kind of regularization is called regularization estimator and its behavior was studied by Li [17] and Speckman [35]. It can be seen from Neubauer [26] that the unique minimizer, say, $x_{\alpha, n, m}^{\delta}$ of problem (5.1) has the form

$$
x_{\alpha, n, m}^{\delta}=x_{\alpha, n, m}^{\delta}(\xi)=G_{\alpha, n, m}\left(y_{\delta}\right),
$$

where

$$
\begin{gathered}
G_{\alpha, n, m}=\left(T_{n, m}^{\#} T_{n, m}+\alpha \mathrm{I}\right)^{-1} T_{n, m}^{\#}=L^{-s}\left(B_{n, m}^{*} B_{n, m}+\alpha \mathrm{I}\right)^{-1} B_{n, m}^{*}, \\
T_{n, m}=Q_{n} A P_{m, s}, \quad B_{n, m}=Q_{n} A P_{m, s} L^{-s},
\end{gathered}
$$

$P_{m, s}$ is the orthogonal projector from $X^{s}$ onto $V_{m}$, and $B^{*}, T^{\#}$ denote the adjoint operators of $B: X \rightarrow X$ and $T: X^{s} \rightarrow X$, respectively. In particular,

$$
T_{n, m}^{\#}=P_{m, s} L^{-2 s} A^{*} Q_{n}, \quad B_{n, m}^{*}=L^{s} P_{m, s} L^{-2 s} A^{*} Q_{n} .
$$

To estimate the performance of the approximating $x_{\alpha, n, m}^{\delta}$, additional properties of the design $\left\{\varphi_{1}, \ldots, \varphi_{n}\right\}$, as well as of the choice of spaces $V_{m}$, are required.

To be precise, we assume that

$$
\left\|\mathrm{I}-Q_{n}\right\|_{Y^{a+t} \rightarrow X} \leq c n^{-(a+t)}, \quad-a \leq t \leq s .
$$

Note that by assumption (2.1) the best possible order of approximation of elements from $Y^{a+t}$ in $Y=X$, using designs of at most $n$ elements, is $n^{-(a+t)}$. We thus assume that this is achieved by our chosen design.

For the projections $P_{m, s}$, projecting onto $V_{m} \subset X^{s}$, we require, as, for example, in Neubauer [26] that

$$
\left\|\mathrm{I}-P_{m, s}\right\|_{X^{a+2 s} \rightarrow X^{s}} \leq c m^{-(a+s)}, \quad-a \leq s .
$$

If $s \geq(\mu-a) / 2$ and (5.4) is fulfilled, then standard interpolation techniques (we refer to Babuška and Aziz [1] for details) yield

$$
\left\|\left(\mathrm{I}-P_{m, s}\right) x_{0}\right\|_{s} \leq c m^{-(\mu-s)}
$$

whenever $x_{0}=A^{-1} y \in X_{R}^{\mu}$, which will be useful below.

Since we have

$$
\begin{aligned}
x_{0}-x_{\alpha, n, m}^{\delta}(\xi) & =x_{0}-G_{\alpha, n, m} A x_{0}-\delta G_{\alpha, n, m} \xi \\
& =\left(\mathrm{I}-G_{\alpha, n, m} A\right) x_{0}-\delta G_{\alpha, n, m} Q_{n} \xi
\end{aligned}
$$

we will estimate both contributions separately. Precisely, we shall estimate

$$
b_{\alpha, n, m}\left(x_{0}\right):=\left\|\left(G_{\alpha, n, m} A-\mathrm{I}\right) x_{0}\right\|, \quad v_{\alpha, n, m}(\xi):=\delta\left\|G_{\alpha, n, m} \xi\right\|
$$

separately. 
Lemma 5.1. Let the assumptions (2.2), (5.3), (5.4) be fulfilled. Assume that for some $\varkappa<1$

$$
\left\|\mathrm{I}-Q_{n}\right\|_{Y^{a} \rightarrow X} \leq \varkappa D^{-1}\left(2^{a} d^{s}\right)^{\frac{1}{a+s}} \alpha^{\frac{a}{2(a+s)}},
$$

where $D, d$ are the constants from (2.2). Then

$$
b_{\alpha, n, m}\left(x_{0}\right) \leq c\left[\alpha^{\frac{\mu}{2(a+s)}}+m^{-s}\left(1+m^{-a} \alpha^{-\frac{a}{2(a+s)}}\right)\left(\alpha^{\frac{\mu-s}{2(a+s)}}+\left\|\left(\mathrm{I}-P_{m, s}\right) x_{0}\right\|_{s}\right)\right] .
$$

Proof. Let $y=A x_{0}$ be the true free term of our equation. Consider the elements $x_{\alpha, n, m}^{0}=G_{\alpha, n, m} y$ and

$$
x_{\alpha, m}^{0}=\left(T_{m}^{\#} T_{m}+\alpha \mathrm{I}\right)^{-1} T_{m}^{\#} y,
$$

where $T_{m}=A P_{m, s}, T_{m}^{\#}=P_{m, s} L^{-2 s} A^{*}$. A close examination of the proof of $[26$, Lemma 3.1] leads to

$$
\left\|\left(T_{m}^{\#} T_{m}+\alpha \mathrm{I}\right)^{-1} T_{m}^{\#}\right\|_{X \rightarrow X} \leq\left(2^{a} d^{s}\right)^{-\frac{1}{a+s}} \alpha^{-\frac{a}{2(a+s)}}
$$

and from Lemma 2.2, Lemma 3.2, ibid., it follows that

$$
\begin{aligned}
& \left\|x_{0}-x_{\alpha, m}^{0}\right\| \\
& \leq c\left[\alpha^{\frac{\mu}{2(a+s)}}+m^{-s}\left(1+m^{-a} \alpha^{-\frac{a}{2(a+s)}}\right)\left(\alpha^{\frac{\mu-s}{2(a+s)}}+\left\|\left(\mathrm{I}-P_{m, s}\right) x_{0}\right\|_{s}\right)\right] .
\end{aligned}
$$

Note that

$$
b_{\alpha, n, m}\left(x_{0}\right)=\left\|x_{0}-x_{\alpha, n, m}^{0}\right\| \leq\left\|x_{0}-x_{\alpha, m}^{0}\right\|+\left\|x_{\alpha, m}^{0}-x_{\alpha, n, m}^{0}\right\| .
$$

Moreover, from (2.2), (5.7), and (5.8) it follows that

$$
\begin{aligned}
\| x_{\alpha, m}^{0} & -x_{\alpha, n, m}^{0} \| \\
& =\left\|\left(T_{m}^{\#} T_{m}+\alpha \mathrm{I}\right)^{-1} T_{m}^{\#}\left(\mathrm{I}-Q_{n}\right) A\left(x_{0}-x_{\alpha, n, m}^{0}\right)\right\| \\
& \leq\left(2^{a} d^{s}\right)^{-\frac{1}{a+s}} \alpha^{-\frac{a}{2(a+s)}}\left\|\mathrm{I}-Q_{n}\right\|_{Y^{a} \rightarrow X}\left\|A\left(x_{0}-x_{\alpha, n, m}^{0}\right)\right\|_{a} \\
& \leq D\left(2^{a} d^{s}\right)^{-\frac{1}{a+s}} \alpha^{-\frac{a}{2(a+s)}}\left\|\mathrm{I}-Q_{n}\right\|_{Y^{a} \rightarrow X} b_{\alpha, n, m}\left(x_{0}\right) \\
& \leq \varkappa b_{\alpha, n, m}\left(x_{0}\right) .
\end{aligned}
$$

Combining (5.9)-(5.11) we obtain the assertion of the lemma.

We mention explicitly that from (5.3) it follows that for

$$
n=\left\lfloor\left(c^{-1} \varkappa D^{-1}\left(2^{a} d^{s}\right)^{\frac{1}{a+s}}\right)^{-\frac{1}{a}} \alpha^{-\frac{1}{2(a+s)}}\right\rfloor,
$$

the assumption (5.7) is fulfilled, where $c$ is the constant from (5.3), and $\lfloor b\rfloor$ is the integer part of $b$.

LEMMA 5.2. Let the assumptions of Lemma 5.1 be fulfilled. If $m \asymp n \asymp \alpha^{-\frac{1}{2(a+s)}}$, then

$$
\left\|G_{\alpha, n, m}\right\|_{X \rightarrow X} \leq c \alpha^{-\frac{a}{2(a+s)}}
$$

where $c$ does not depend on $\alpha, n, m$. 
Proof. It is well known that for an arbitrary compact operator $B$ from $X$ to $X$

$$
\left\|\left(B^{*} B+\alpha \mathrm{I}\right)^{-1} B^{*}\right\|_{X \rightarrow X} \leq \frac{1}{2 \sqrt{\alpha}}, \quad\left\|B\left(B^{*} B+\alpha \mathrm{I}\right)^{-1} B^{*}\right\|_{X \rightarrow X} \leq 1 .
$$

In particular we have for any $f \in X$ the bound

$$
\begin{aligned}
\left\|G_{\alpha, n, m} f\right\|_{s} & =\left\|L^{s} G_{\alpha, n, m} f\right\| \\
& =\left\|\left(B_{n, m}^{*} B_{n, m}+\alpha \mathrm{I}\right)^{-1} B_{n, m}^{*} f\right\| \leq \frac{1}{2 \sqrt{\alpha}}\|f\| .
\end{aligned}
$$

Moreover, from (2.2) and (5.13) it follows that

$$
\begin{aligned}
\left\|G_{\alpha, n, m} f\right\|_{-a} & \leq d^{-1}\left\|A G_{\alpha, n, m} f\right\| \\
& \leq d^{-1}\left\|B_{n, m}\left(B_{n, m}^{*} B_{n, m}+\alpha \mathrm{I}\right)^{-1} B_{n, m}^{*} f\right\| \\
& +d^{-1}\left\|\left(A L^{-s}-B_{n, m}\right)\left(B_{n, m}^{*} B_{n, m}+\alpha \mathrm{I}\right)^{-1} B_{n, m}^{*} f\right\| \\
& \leq d^{-1}\left(1+\frac{1}{2 \sqrt{\alpha}}\left\|A L^{-s}-B_{n, m}\right\|_{X \rightarrow X}\right)\|f\| .
\end{aligned}
$$

Now we derive an estimate for $\left\|A L^{-s}-B_{n, m}\right\|_{X \rightarrow X}$ :

$$
\begin{aligned}
\left\|A L^{-s}-B_{n, m}\right\|_{X \rightarrow X} & \leq\left\|A L^{-s}-A P_{m, s} L^{-s}\right\|_{X \rightarrow X} \\
& +\left\|A P_{m, s} L^{-s}-Q_{n} A P_{m, s} L^{-s}\right\|_{X \rightarrow X} .
\end{aligned}
$$

By (2.2) and (5.4) we can continue

$$
\begin{aligned}
\left\|A L^{-s}-A P_{m, s} L^{-s}\right\|_{X \rightarrow X} & \asymp\left\|\left(\mathrm{I}-P_{m, s}\right) L^{-s}\right\|_{X \rightarrow X}{ }^{-a} \\
& =\left\|L^{s} L^{-s-a}\left(\mathrm{I}-P_{m, s}\right) L^{-s}\right\|_{X \rightarrow X} \\
& =\left\|L^{-s-a}\left(\mathrm{I}-P_{m, s}\right)\right\|_{X^{s} \rightarrow X^{s}} \\
& =\left\|\left(\mathrm{I}-P_{m, s}\right)\right\|_{X^{2 s+a} \rightarrow X^{s}} \\
& \leq c m^{-(s+a)} \asymp n^{-(s+a)} .
\end{aligned}
$$

(Note that $L^{-\nu}: X^{t} \rightarrow X^{t}$ is self-adjoint for $\nu \geq t$ ). Further, using (2.2) and (5.3) we find

$$
\begin{aligned}
\left\|A P_{m, s} L^{-s}-Q_{n} A P_{m, s} L^{-s}\right\|_{X \rightarrow X} & \leq\left\|\mathrm{I}-Q_{n}\right\|_{Y^{a+s} \rightarrow X}\left\|A P_{m, s} L^{-s}\right\|_{X \rightarrow Y^{a+s}} \\
& \leq c n^{-(a+s)}\left\|P_{m, s} L^{-s}\right\|_{X \rightarrow X^{s}} \\
& \asymp n^{-(a+s)}\left\|P_{m, s}\right\|_{X^{s} \rightarrow X^{s}} \leq c n^{-(a+s)} .
\end{aligned}
$$

Then for $m \asymp n \asymp \alpha^{-\frac{1}{2(a+s)}}$

$$
\left\|G_{\alpha, n, m} f\right\|_{-a} \leq c\|f\|
$$

Interpolation together with (5.14) yields

$$
\left\|G_{\alpha, n, m} f\right\|_{0} \leq\left\|G_{\alpha, n, m} f\right\|_{s}^{\frac{a}{a+s}}\left\|G_{\alpha, n, m} f\right\|_{-a}^{\frac{s}{a+s}} \leq c \alpha^{-\frac{a}{2(a+s)}}\|f\| .
$$

The lemma is proved. 
THEOREM 5.3. Let the assumptions of Lemma 5.1 be fulfilled and suppose that the unknown solution belongs to $X_{R}^{\mu}$. Further assume $s \geq \max \{\nu,(\mu-a) / 2\}$.

If we choose $n$ as in (5.12), $\alpha \asymp \delta^{\frac{2(a+s)}{\mu+a+1 / 2}}$, and $m \asymp n \asymp \delta^{-\frac{1}{\mu+a+1 / 2}}$, then for Gaussian white noise satisfying (1.6) and $\nu \in[-a, \mu]$ we have

$$
\left(\mathbf{E}\left\|x_{0}-x_{\alpha, n, m}^{\delta}(\xi)\right\|_{\nu}^{2}\right)^{1 / 2} \leq c \delta^{\frac{\mu-\nu}{\mu+a+1 / 2}} .
$$

The constant above in estimate (5.15) does not depend on $\alpha, \delta, m$, and $n$.

Proof. The proof is carried out in two steps. We first provide the required estimates for $\nu=0$.

For any realization of $\xi$ it follows from Lemmas 5.1 and 5.2 and (5.5) that

$$
\begin{aligned}
\left\|x_{0}-x_{\alpha, n, m}(\xi)\right\| & \leq b_{\alpha, n, m}\left(x_{0}\right)+v_{\alpha, n, m}(\xi) \\
& \leq c \alpha^{\frac{\mu}{2(a+s)}}+\delta\left\|G_{\alpha, n, m}\right\|_{X \rightarrow X}\left\|Q_{n} \xi\right\| \\
& \leq c\left[\alpha^{\frac{\mu}{2(a+s)}}+\delta \alpha^{-\frac{a}{2(a+s)}}\left\|Q_{n} \xi\right\|\right] .
\end{aligned}
$$

For white noise, property (1.6) implies

$$
\mathbf{E}\left\|Q_{n} \xi\right\|^{2}=\sum_{i=1}^{n} \mathbf{E}\left|\left\langle\varphi_{i}, \xi\right\rangle\right|^{2}=\sum_{i=1}^{n}\left\|\varphi_{i}\right\|^{2}=n \asymp \delta^{-\frac{1}{\mu+a+1 / 2}},
$$

and we can continue (5.16) to arrive at

$$
\begin{aligned}
\mathbf{E}\left\|x_{0}-x_{\alpha, n, m}^{\delta}(\xi)\right\|^{2} & \leq c\left[\alpha^{\frac{\mu}{a+s}}+\delta^{2} \alpha^{-\frac{a}{a+s}} n\right] \\
& \leq c\left[\alpha^{\frac{\mu}{(a+s)}}+\delta^{2} \alpha^{-\frac{2 a+1}{2(a+s)}}\right] \leq c \delta^{\frac{2 \mu}{\mu+a+1 / 2}} .
\end{aligned}
$$

This is estimate (5.15) for $\nu=0$.

We now turn to the general case $\nu \neq 0$. We note that if we let $x_{\alpha, n, m}^{\delta, \nu}(\xi):=$ $L^{\nu} x_{\alpha, n, m}^{\delta}(\xi)$, then this is the unique minimizer of the functional

$$
\left\|Q_{n} A L^{-\nu} x-Q_{n} y_{\delta}\right\|^{2}+\alpha\|x\|_{s_{\nu}}^{2}
$$

where we let $s_{\nu}:=s-\nu$ on the space $V_{m, \nu}:=L^{\nu} V_{m} \subset X^{s-\nu}$. Moreover, the orthogonal projector $P_{m, s-\nu}:=L^{\nu} P_{m, s} L^{-\nu}$ in $X^{s-\nu}$ onto this subspace obeys the corresponding variant of (5.4) with respective $a_{\nu}:=a+\nu$, since

$$
\begin{aligned}
\left\|\mathrm{I}-P_{m, s-\nu}\right\|_{X^{a_{\nu}+2 s_{\nu}} \rightarrow X^{s_{\nu}}} & =\left\|\mathrm{I}-L^{\nu} P_{m, s} L^{-\nu}\right\|_{X^{a_{\nu}+2 s_{\nu}} \rightarrow X^{s_{\nu}}} \\
& =\left\|\mathrm{I}-P_{m, s}\right\|_{X^{a+2 s} \rightarrow X^{s}} \\
& \leq \mathrm{cm}^{-(a+s)}=\mathrm{cm}^{-\left(a_{\nu}+s_{\nu}\right)} .
\end{aligned}
$$

Moreover, the operator $A_{\nu}:=A L^{-\nu}$ obeys condition (2.2) with $a_{\nu}$, and finally $x_{0}^{\nu}:=$ $L^{\nu} x_{0} \subset X_{R}^{\mu-\nu}$. Thus, using the estimates of the first part of the proof for $A_{\nu}, a_{\nu}, \mu_{\nu}:=$ $\mu-\nu, s_{\nu}$ and projection $P_{m, s_{\nu}}$ we obtain for $\alpha \asymp \delta^{\frac{2\left(a_{\nu}+s_{\nu}\right)}{a+\mu+1 / 2}}$ that

$$
\begin{aligned}
\left(\mathbf{E}\left\|x_{0}-x_{\alpha, n, m}^{\delta}(\xi)\right\|_{\nu}^{2}\right)^{1 / 2} & =\left(\mathbf{E}\left\|L^{\nu} x_{0}-L^{\nu} x_{\alpha, n, m}^{\delta}(\xi)\right\|^{2}\right)^{1 / 2} \\
& =\left(\mathbf{E}\left\|x_{0}^{\nu}-x_{\alpha, n, m}^{\delta, \nu}(\xi)\right\|^{2}\right)^{1 / 2} \\
& \leq c \delta^{\frac{\mu_{\nu}}{\mu_{\nu}+a_{\nu}+1 / 2}}=c \delta^{\frac{\mu-\nu}{\mu+a+1 / 2}} .
\end{aligned}
$$

This concludes the proof for $\nu \neq 0$ and of the theorem. 
We mention explicitly that the same method was chosen to yield the optimal approximation in all spaces $X^{\nu}$. This will be optimal as long as the parameter $s$ is chosen large enough.

THEOREM 5.4. Let the assumptions (2.2), (2.1), and (2.8) be fulfilled. Then for Gaussian white noise satisfying (1.6) and for $\nu \in[-a, \mu]$ the following asymptotics holds true:

$$
N^{\operatorname{ran}}\left(A, \mathcal{U}, X_{R}^{\mu}, \delta\right) \asymp \delta^{-\frac{1}{\mu+a+1 / 2}} .
$$

The optimal order of information complexity is achieved by Tikhonov regularization (5.1) if only the design obeys (5.3).

Proof. Let $n=n(\delta)$ be chosen as in (5.12) for $\alpha \asymp \delta^{\frac{2(a+s)}{\mu+a+1 / 2}}$, i.e., $n(\delta) \asymp \delta^{-\frac{1}{\mu+a+1 / 2}}$. From Theorem 5.3 we draw that for $n \geq n(\delta)$ the following estimate holds true:

$$
\begin{aligned}
e^{r a n}\left(A, \mathcal{U}, X_{R}^{\mu}, \delta\right) & \leq r_{n}^{r a n}\left(A, X_{R}^{\mu}, \delta\right) \\
& \leq r_{n(\delta)}^{r a n}\left(A, X_{R}^{\mu}, \delta\right) \leq c \delta^{\frac{\mu-\nu}{\mu+a+1 / 2}} .
\end{aligned}
$$

Since by Proposition 3.1

$$
\delta^{\frac{\mu-\nu}{\mu+a+1 / 2}} \asymp \mathcal{E}^{r a n}\left(A, X_{R}^{\mu}, \delta\right) \leq e^{r a n}\left(A, \mathcal{U}, X_{R}^{\mu}, \delta\right),
$$

we conclude that actually $e^{r a n}\left(A, \mathcal{U}, X_{R}^{\mu}, \delta\right) \asymp \delta^{\frac{\mu-\nu}{\mu+a+1 / 2}}$ such that for some sufficiently large $C$ we have

$$
N^{r a n}\left(A, \mathcal{U}, X_{R}^{\mu}, \delta\right) \leq n(\delta) \asymp \delta^{-\frac{1}{\mu+a+1 / 2}} .
$$

On the other hand, using Corollary 1 and estimate (5.18) we deduce

$$
\begin{aligned}
&\{n, \quad\left.r_{n}^{r a n}\left(A, X_{R}^{\mu}, \delta\right) \leq C e^{r a n}\left(A, \mathcal{U}, X_{R}^{\mu}, \delta\right)\right\} \\
& \subset\left\{n, \quad r_{n}^{r a n}\left(A, X_{R}^{\mu}, \delta\right) \leq C \delta^{\frac{\mu-\nu}{\mu+a+1 / 2}}\right\} \\
& \subset\left\{n, \quad n^{-(\mu-\nu)} \leq \tilde{C} \delta^{\frac{\mu-\nu}{\mu+a+1 / 2}}\right\} .
\end{aligned}
$$

Hence

$$
N^{r a n}\left(A, \mathcal{U}, X_{R}^{\mu}, \delta\right) \geq \inf \left\{n, n^{-(\mu-\nu)} \leq \tilde{C} \delta^{\frac{\mu-\nu}{\mu+a+1 / 2}}\right\} \asymp \delta^{-\frac{1}{\mu+a+1 / 2}},
$$

which concludes the proof of the theorem.

Remark 2. As already mentioned in section 4, previous study of Tikhonov regularization in Hilbert scales was restricted to the semidiscrete setup; see [12]. There the approximate solution of (1.1) is determined by

$$
x_{\alpha, m}^{\delta}:=\operatorname{argmin}\left\{\left\|A x-y_{\delta}\right\|^{2}+\alpha\|x\|_{s}^{2}, \quad x \in V_{m} \subset X^{s}\right\},
$$

which can be represented also as $x_{\alpha, m}^{\delta}=\left(\alpha \mathrm{I}+T_{m}^{\#} T_{m}\right)^{-1} T_{m}^{\#} y_{\delta}$, where $T_{m}:=A P_{m, s}$, and $T_{m}^{\#}:=P_{m, s} L^{-2 s} A^{*}$; cf. (5.2). From this representation it follows that in order to construct $x_{\alpha, m}^{\delta}$ one needs the following discrete information of the data, namely,

$$
y_{\delta, i}=\left\langle\psi_{i}, A^{*} y_{\delta}\right\rangle=\left\langle y_{\delta}, A \psi_{i}\right\rangle, i=1,2, \ldots, m,
$$

where $\left\{\psi_{i}\right\}_{i=1}^{m}$ is some orthonormal basis of $V_{m} \subset X^{s}$. Now this immediately implies that in the version of Tikhonov regularization just described, the design cannot be chosen independently of the operator $A$, which is a drawback, since it is often given, for example, as histogram (1.5). 
6. Self-regularization properties of projection methods. Two-sided discretization of the least-square method. As we have already discussed in section 4 , the most prominent projection scheme with self-regularization properties is the least-square method, where we agree to consider as regularized solution of (1.1) any element $x_{m}^{\delta}$, which minimizes

$$
x_{m}^{\delta}:=\operatorname{argmin}\left\{\left\|A x-y_{\delta}\right\|, \quad x \in V_{m}\right\} .
$$

The least-square method in the form as given above has extensively been studied by Natterer [24], Vaŭnikko and Khyamarik [39], Louis [18], and Dicken and Maass [8].

As it turns out, this leads again to a one-sided discretization, because (6.1) is equivalent to Gauss symmetrization of the one-sided discretized equation

$$
A P_{m} y=y_{\delta} .
$$

More precisely, $x_{m}^{\delta}$ solves the equation

$$
P_{m} A^{*} A P_{m} x=P_{m} A^{*} y_{\delta}
$$

and uses the discretized information of the form

$$
y_{\delta, i}=\left\langle\psi_{i}, A^{*} y_{\delta}\right\rangle=\left\langle A \psi_{i}, y_{\delta}\right\rangle, \quad i=1, \ldots, m,
$$

where again $\left\{\psi_{i}\right\}_{i=1}^{m}$ is an orthonormal basis of $V_{m} \subset X$. Thus in its standard form (6.1), (6.3), the least-square method in most cases cannot use given observations, as, for example, in (1.2), independently of the operator $A$. Of course, this can be overcome when using instead of (6.2) the equation

$$
A P_{m} x=Q_{n} y_{\delta}
$$

and obtaining the regularized solution $\hat{x}_{n, m}^{\delta}$ from the resulting symmetrization

$$
P_{m} A^{*} A P_{m} x=P_{m} A^{*} Q_{n} y_{\delta}
$$

being equivalent to

$$
\hat{x}_{n, m}^{\delta}:=\operatorname{argmin}\left\{\left\|A x-Q_{n} y \delta\right\|, \quad x \in V_{m}\right\} .
$$

However, this is inconvenient, since it requires two different discretizations of the operator $A$, instead of the simple projection scheme

$$
Q_{n} A P_{m} x=Q_{n} y_{\delta} .
$$

For this projection scheme (6.5) a little more effort has to be made in order to even assume solvability. In fact, even if the original operator $A$ was invertible, it may occur that the sections $P_{n} A P_{n}$ are not (see Böttcher et al. [3] for more details). However, after Gauss symmetrization of (6.5), by which we mean turning to

$$
P_{m} A^{*} Q_{n} A P_{m} x=P_{m} A^{*} Q_{n} y_{\delta},
$$

there is always a solution, say, $x_{n, m}^{\delta}$. This, however, need not be unique. The uniqueness can be achieved only if $\operatorname{ker}\left(Q_{n} A P_{m}\right) \cap \operatorname{Im}\left(P_{m}\right)=\{0\}$, which was observed by Vaŭnikko and Khyamarik [39]. This in turn can be achieved when the operators 
$Q_{n} A P_{m}$ obey the following stability property: there is a constant $c_{s}>0$ such that for all $u \in V_{m}$ we have

$$
c_{s}\left\|P_{m} u\right\|_{\nu} \leq\left\|Q_{n} A P_{m} u\right\|_{\nu+a}
$$

see Lemma 6.2 below.

We now turn to the assumptions, both of Jackson and Bernstein type, made on the projections $P_{m}$ onto the subspace $V_{m}$ and $Q_{n}$ corresponding to the design $\varphi=\left\{\varphi_{1}, \ldots, \varphi_{n}\right\}$ in order to make the projection scheme efficient. Precisely we assume that there is a positive $s>0$, and there are constants $b, q>1$ such that $V_{m} \subset X^{s+\mu}$ and $P_{m}$ obeys for $\nu \in[-a, \mu]$ the estimate

$$
\left\|\mathrm{I}-P_{m}\right\|_{X^{\mu} \rightarrow X^{\nu}} \leq q m^{-(\mu-\nu)}
$$

and

$$
\left\|P_{m} u\right\|_{s+\nu} \leq b m^{s}\left\|P_{m} u\right\|_{\nu}, \quad \nu \in[-a, \mu]
$$

The projections $Q_{n}$ satisfy

$$
\left\|\mathrm{I}-Q_{n}\right\|_{Y^{s+\nu+a} \rightarrow Y^{\nu+a}} \leq q n^{-s}
$$

and also

$$
\left\|\mathrm{I}-Q_{n}\right\|_{Y^{\mu+a} \rightarrow X} \leq q n^{-(\mu+a)} .
$$

Moreover, we assume that $\operatorname{Im}\left(Q_{n}\right) \subset Y^{\nu+a}$ and

$$
\left\|Q_{n} u\right\|_{\nu+a} \leq b n^{\nu+a}\left\|Q_{n} u\right\| .
$$

Assumptions of this kind were also made in [7]. Under the above assumptions the following bound for the approximate solution $\hat{x}_{n, m}^{\delta}$, as defined through (6.4), has been proven; see, e.g., [18, Satz 4.5.6]

Proposition 6.1. Assume that assumptions (6.7) and (6.8) hold. Then for operators $A$ satisfying (2.2) and for $x_{0}:=A^{-1} y \in X^{\mu}$ we have

$$
\left\|x_{0}-\hat{x}_{n, m}^{\delta}\right\|_{\nu} \leq c\left(\left\|\left(\mathrm{I}-P_{m}\right) x_{0}\right\|_{\nu}+m^{\nu+a}\left\|y-Q_{n} y_{\delta}\right\|\right) .
$$

As mentioned before, a certain stability is required for self-regularization. Usually this kind of stability is assumed to be fulfilled for a given projection scheme; see, e.g., [4], or it can be deduced from properties of the operator $A$; here we refer to [33], where Symm's equation was studied. If we allow $m \neq n$, then we may deduce stability from our assumptions made above.

Lemma 6.2. Assume (2.2) and (6.8), (6.9) to hold. Then there is $0<c_{0}<1$ such that for sufficiently large $n$ and $m=\left\lfloor c_{0} n\right\rfloor$, the integer part of $c_{0} n$, stability

$$
c_{s}\left\|P_{m} u\right\|_{\nu} \leq\left\|Q_{n} A P_{m} u\right\|_{\nu+a}
$$

holds.

In particular, for $c_{0}:=(d /(2 D q b))^{1 / s}$ we have $(6.12)$ with $c_{s}=d / 2$.

Proof. We derive from assumption (2.2) that

$$
d\left\|P_{m} u\right\|_{\nu} \leq\left\|A P_{m} u\right\|_{\nu+a} \leq\left\|\left(\mathrm{I}-Q_{n}\right) A P_{m} u\right\|_{\nu+a}+\left\|Q_{n} A P_{m} u\right\|_{\nu+a}
$$


for every $u \in V_{m}$. Now using (6.8) and (6.9), this yields

$$
\begin{aligned}
\left\|\left(\mathrm{I}-Q_{n}\right) A P_{m} u\right\|_{\nu+a} & \leq q n^{-s}\left\|A P_{m} u\right\|_{s+\nu+a} \\
& \leq D q n^{-s}\left\|P_{m} u\right\|_{s+\nu} \leq D q b(m / n)^{s}\left\|P_{m} u\right\|_{\nu},
\end{aligned}
$$

which implies

$$
\left[d-D q b(m / n)^{s}\right]\left\|P_{m} u\right\|_{\nu} \leq\left\|Q_{n} A P_{m} u\right\|_{\nu+a} .
$$

It is now easy to derive the remaining assertion for $c_{0}:=(d /(2 D q b))^{1 / s}$.

We turn to the main result of this section.

TheOREm 6.3. Assume that the operator A obeys (2.2) and that the assumptions (6.7)-(6.11) are fulfilled. In the white noise setting, for $x_{0}=A^{-1} y \in X_{R}^{\mu}$ and $m=\left\lfloor c_{0} n\right\rfloor \asymp \delta^{-1 /(\mu+a+1 / 2)}$, where $c_{0}$ is from Lemma 6.2 , there is a constant $c$, for which we can bound the error of the approximate solution obtained from (6.6) by

$$
\left(\mathbf{E}\left\|x_{0}-x_{n, m}^{\delta}\right\|_{\nu}^{2}\right)^{1 / 2} \leq c \delta^{\frac{\mu-\nu}{\mu+a+1 / 2}} .
$$

Proof. Of course,

$$
\left\|x_{0}-x_{n, m}^{\delta}\right\|_{\nu} \leq\left\|x_{0}-\hat{x}_{n, m}^{\delta}\right\|_{\nu}+\left\|\hat{x}_{n, m}^{\delta}-x_{n, m}^{\delta}\right\|_{\nu} .
$$

The first norm can further be estimated using Proposition 6.1 and assumptions (6.7), (6.10). We derive for $m=\left\lfloor c_{0} n\right\rfloor$ the estimate

$$
\begin{aligned}
\left\|x_{0}-\hat{x}_{n, m}^{\delta}\right\|_{\nu} & \leq c\left(\left\|\left(\mathrm{I}-P_{m}\right) x_{0}\right\|_{\nu}+m^{\nu+a}\left\|\left(\mathrm{I}-Q_{n}\right) y\right\|+m^{\nu+a} \delta\left\|Q_{n} \xi\right\|\right) \\
& \leq c\left(m^{-(\mu-\nu)}\left\|x_{0}\right\|_{\mu}+m^{\nu+a} m^{-(\mu+a)}\|y\|_{\mu+a}+m^{\nu+a} \delta\left\|Q_{n} \xi\right\|\right) \\
& \leq c\left(m^{-(\mu-\nu)}\left\|x_{0}\right\|_{\mu}+m^{\nu+a} \delta\left\|Q_{n} \xi\right\|\right) .
\end{aligned}
$$

If we now use $\hat{x}_{n, m}^{\delta}, x_{n, m}^{\delta} \in V_{m}$, then we can derive from (6.11) and (6.12) the following chain of estimates for the second summand in (6.13):

$$
\begin{aligned}
\left\|\hat{x}_{n, m}^{\delta}-x_{n, m}^{\delta}\right\|_{\nu} & \leq c_{s}^{-1}\left\|Q_{n} A P_{m}\left(\hat{x}_{n, m}^{\delta}-x_{n, m}^{\delta}\right)\right\|_{\nu+a} \\
& \leq c_{s}^{-1} b n^{\nu+a}\left\|Q_{n} A P_{m}\left(\hat{x}_{n, m}^{\delta}-x_{n, m}^{\delta}\right)\right\| \\
& \leq c n^{\nu+a}\left(\left\|Q_{n} A P_{m} x_{n, m}^{\delta}-Q_{n} y_{\delta}\right\|+\left\|Q_{n} A P_{m} \hat{x}_{n, m}^{\delta}-Q_{n} y_{\delta}\right\|\right) .
\end{aligned}
$$

Now $x_{n, m}^{\delta}$ from (6.6) has a representation as

$$
x_{n, m}^{\delta}=\operatorname{argmin}\left\{\left\|Q_{n} A P_{m} x-Q_{n} y_{\delta}\right\|, \quad x \in V_{m}\right\} .
$$

Using (6.7) we can continue

$$
\begin{aligned}
\left\|Q_{n} A P_{m} x_{n, m}^{\delta}-Q_{n} y_{\delta}\right\| & \leq\left\|Q_{n} A P_{m} x_{0}-Q_{n} y_{\delta}\right\| \\
& \leq\left\|Q_{n} A x_{0}-Q_{n} y_{\delta}\right\|+\left\|Q_{n} A\left(\mathrm{I}-P_{m}\right) x_{0}\right\| \\
& \leq \delta\left\|Q_{n} \xi\right\|+D\left\|\left(\mathrm{I}-P_{m}\right) x_{0}\right\|_{-a} \\
& \leq \delta\left\|Q_{n} \xi\right\|+c m^{-(\mu+a)}\left\|x_{0}\right\|_{\mu} .
\end{aligned}
$$


Moreover, from the definition of $\hat{x}_{n, m}^{\delta}$ in (6.4) and assumption (6.10) we conclude

$$
\begin{aligned}
\left\|Q_{n} A P_{m} \hat{x}_{n, m}^{\delta}-Q_{n} y_{\delta}\right\| & \leq\left\|A P_{m} \hat{x}_{n, m}^{\delta}-Q_{n} y_{\delta}\right\| \leq\left\|A P_{m} x_{0}-Q_{n} y_{\delta}\right\| \\
& \leq\left\|A\left(\mathrm{I}-P_{m}\right) x_{0}\right\|+\left\|\left(\mathrm{I}-Q_{n}\right) y\right\|+\delta\left\|Q_{n} \xi\right\| \\
& \leq c\left(m^{-(\mu+a)}\left\|x_{0}\right\|_{\mu}+\delta\left\|Q_{n} \xi\right\|\right) .
\end{aligned}
$$

Thus for $m=\left\lfloor c_{0} n\right\rfloor$ the previous estimates, after being inserted into (6.13) yield

$$
\left\|x_{0}-x_{n, m}^{\delta}\right\|_{\nu} \leq c\left(m^{-(\mu-\nu)}\left\|x_{0}\right\|_{\mu}+\delta m^{\nu+a}\left\|Q_{n} \xi\right\|\right)
$$

Since, as in the proof of Theorem 5.3, $\mathbf{E}\left\|Q_{n} \xi\right\|^{2}=n$, we deduce for $n \asymp m \asymp \delta^{-\frac{1}{\mu+a+1 / 2}}$

$$
\left(\mathbf{E}\left\|x_{0}-x_{n, m}^{\delta}\right\|_{\nu}^{2}\right)^{1 / 2} \leq c\left(m^{-(\mu-\nu)}\left\|x_{0}\right\|_{\mu}+\delta m^{\nu+a+1 / 2}\right) \leq c \delta^{\frac{\mu-\nu}{\mu+a+1 / 2}}
$$

which proves this theorem.

Remark 3. We mention that even for deterministic noise $\xi,\|\xi\|_{X} \leq 1$, the analogues of Theorems 5.3, 5.4, and 6.3 are new. Since the proofs are very similar, we only briefly formulate the corresponding statements. In the deterministic case, under assumptions $(2.1),(2.2)$ and for $\nu \in[-a, \mu]$, we have

$$
N^{\operatorname{det}}\left(A, \mathcal{U}, X_{R}^{\mu}, \delta\right) \asymp \delta^{-\frac{1}{\mu+a}}
$$

The optimal order of information complexity is achieved within the framework of selfregularization scheme $(6.5),(6.6)$ for $m=\left\lfloor c_{0} n\right\rfloor \asymp \delta^{-\frac{1}{\mu+a}}$ if the assumptions (6.7)(6.9) and (6.11) are fulfilled. We note that for deterministic noise, condition (6.10) is not required for self-regularization.

This order is also achieved by Tikhonov regularization if only the design obeys (5.3), $n$ is chosen as in (5.12), $\alpha \asymp \delta^{2(a+s) /(\mu+a)}$, and $m \asymp n$.

7. Application to Abel's equation. There are various situations where the results of the previous sections apply to some given problem. Below we will demonstrate how the techniques of the previous sections apply to Abel's equation as introduced in Example 2, section 2, although not all of the assumptions, in particular on the required approximation properties, are fulfilled. It will be important to assume that the design is given beforehand in the form of histograms (1.5). Noisy Abel's equation (1.1), with operator (2.4) arises from a diverse range of applications in the physical sciences and in stereological microscopy. Some pertinent references are Nychka and Cox [29], Johnstone and Silverman [15], and Donoho [9].

Let us discuss some relations to the above mentioned paper [29] in more detail. These authors study this problem in the context of the classical Sobolev spaces (with boundary conditions). As a consequence the error bounds are not given in $L_{2}(0,1)$ but can be derived only in some interpolation norm as presented in Theorem 2.1 and result in final estimates of the residual error [29, Theorem 1.1 and Corollary 1.1]. Also, the authors aim at studying the approximate solution based on histogram observations. Since their initial assumption is based on observing noisy function values, this causes extra work for adapting this particular problem to the assumptions of the paper.

Our study uses histogram observations directly, since these are a particular instance of orthogonal design, consisting of piecewise constant functions. However, to fit the problem to a Hilbert scale, we switch to other Hilbert spaces other than the 
classical Sobolev ones. This allows us to obtain direct error estimates, which prove to be optimal. We postpone further discussion to Remark 4.

As already mentioned in Example 2, we study the problem in the Hilbert scales generated by $L:=\left(A^{*} A\right)^{-1}, L_{1}:=\left(A A^{*}\right)^{-1}$ such that assumption (2.2) is fulfilled with $a=1 / 2$. We shall restrict ourselves to the case where the exact solution belongs to $X_{R}^{1}$; thus $\mu=1$. This is the maximal a priori smoothness, when we can satisfy the required approximation properties within the subspace of piecewise constant functions. In this case, the exact solution admits a representation

$$
x_{0}=A^{*} A g_{0}
$$

for some $g_{0} \in L_{2}(0,1), \quad\left\|g_{0}\right\| \leq R$. Using Corollary 1 in Samko [31] we obtain the following representations:

$$
A f(t)=A^{*} V f(t),
$$

where $A^{*}$ is given by (2.5) and the operator

$$
V f(t):=\frac{1}{\pi \sqrt{t}} \int_{0}^{1} \frac{\sqrt{\tau} f(\tau)}{\tau-t} d \tau
$$

acts boundedly from $L_{2}(0,1)$ into the space $L_{2,2 \varepsilon}(0,1)$ of functions that are squaresummable on $(0,1)$ with weight $t^{2 \varepsilon}$, where $\varepsilon>0$ is arbitrarily small. This means that for any $f \in L_{2}(0,1)$ there exists $f_{\varepsilon} \in L_{2}(0,1)$ such that

$$
V f(t)=t^{-\varepsilon} f_{\varepsilon}(t) \quad \text { and } \quad\left\|f_{\varepsilon}\right\| \leq c\|f\| .
$$

Then (7.1) and (7.2) together with the semigroup property of fractional integration imply that

$$
x_{0}(t)=A^{*} A g_{0}(t)=A^{*} A^{*} V g_{0}(t)=\int_{0}^{t} \tau^{-\varepsilon} g_{0, \varepsilon}(\tau) d \tau .
$$

Thus $x_{0}$ has derivative $x_{0}^{\prime} \in L_{2,2 \varepsilon}(0,1)$ for any small $\varepsilon>0$. In terms of the modulus of continuity we can estimate the smoothness of the solution $x_{0}$ by

$$
\omega_{2}\left(x_{0}, h\right):=\sup _{0<t \leq h}\left(\int_{0}^{1-t}\left|x_{0}(t+\tau)-x_{0}(\tau)\right|^{2} d \tau\right)^{1 / 2}=O\left(h^{1-\varepsilon}\right)
$$

for any small $\varepsilon>0$. obey

As in Nychka and Cox [29] we will assume that the bin limits of histograms (1.5)

$$
\max _{j=1,2, \ldots, n}\left|u_{j, n}-u_{j-1, n}\right| \asymp n^{-1} .
$$

We represent (1.5) in the form (1.3), where $Q_{n}$ is the orthogonal projector on the subspace of piecewise constant functions having discontinuities at the points $\left\{u_{i, n}\right\}$.

Finally we agree to measure the error in $X=L_{2}(0,1)$. Note that under these assumptions the histogram design does not meet conditions (6.9) and (6.11), which are the keys for self-regularization. Indeed, assumption (6.9) would require that $\operatorname{Im}\left(Q_{n}\right) \subset Y^{1 / 2}=\operatorname{Im}\left(A A^{*}\right)^{1 / 2} \subseteq \operatorname{Im}(A)$ (the last relation follows from the singular value decomposition of the compact operator). However, for the constant function 
$\varphi_{0}(t) \equiv 1$, which is certainly in the range of $Q_{n}$, this would imply the existence of a function $v_{0} \in L_{2}(0,1)$, for which

$$
A v_{0}(t)=\frac{1}{\sqrt{\pi}} \int_{t}^{1} \frac{v_{0}(\tau)}{\sqrt{\tau-t}} d \tau=\varphi_{0}(t)=1 .
$$

We can use the inversion formula to see

$$
v_{0}(t)=-\frac{1}{\sqrt{\pi}} \frac{d}{d t} \int_{t}^{1} \frac{d \tau}{\sqrt{\tau-t}}=\frac{1}{\sqrt{\pi(1-t)}} .
$$

It is immediate that this function cannot belong to $L_{2}(0,1)$, which in turn implies $\operatorname{Im}\left(Q_{n}\right) \not \subset Y^{1 / 2}$. Therefore the histogram design does not give rise to selfregularization and we are led to apply Tikhonov regularization based on the de$\operatorname{sign}(1.5)$.

We first observe that Abel's integral operator $A$ acts continuously from $L_{2}(0,1)$ to the space $H_{2}^{1 / 2} \subset L_{2}(0,1)$ of functions for which

$$
\|f\|_{H_{2}^{1 / 2}}:=\|f\|_{L_{2}}+\sup _{0<h<1} \frac{\omega_{2}(f, h)}{\sqrt{h}}<\infty ;
$$

we refer to [32, Thm. 14.2]. On the other hand, using the singular value decomposition one can see that any function $f \in Y^{1 / 2} \subseteq \operatorname{Im}\left[\left(A A^{*}\right)^{1 / 2}\right]$ has the representation $f=A g$, where $\|f\|_{1 / 2}=\|g\|_{L_{2}}$. Then

$$
\begin{aligned}
\left\|\left(I-Q_{n}\right) f\right\|_{L_{2}} & \leq c \omega_{2}\left(f, n^{-1}\right)=c \omega_{2}\left(A g, n^{-1}\right) \\
& \leq c n^{-1 / 2}\|A\|_{L_{2} \rightarrow H_{2}^{1 / 2}}\|g\|_{L_{2}} \leq c n^{-1 / 2}\|f\|_{1 / 2} .
\end{aligned}
$$

This is just condition (5.3) for $t=0$. A straightforward application of Theorem 5.3 in the present case requires us to take $s \geq \frac{\mu-a}{2}=\frac{1}{4}$. It is inconvenient to use Tikhonov method (5.1), (5.2) when $s$ is a fraction. However, for $s=1$ the condition (5.3) breaks down for our case, because using $Q_{n}$ we cannot obtain an accuracy being superior to $O\left(n^{-1}\right)$. Therefore we let $s=0$. Then condition $s \geq \frac{\mu-a}{2}$ is violated, but if we only slightly change the value $m\left(m=\alpha^{-\frac{1}{1-\varepsilon}}\right.$ instead of $\left.m=\alpha^{-1}\right)$, then estimate (7.5) allows us to obtain the same order of accuracy as in Theorem 5.3.

Since $s=0$, we let $P_{m, 0}=Q_{m}$, the orthogonal projector like $Q_{n}$ but corresponding to $m$ bins. Estimate (7.5) implies

$$
\left\|\left(\mathrm{I}-P_{m, 0}\right) x_{0}\right\|_{0}=\left\|\left(\mathrm{I}-Q_{m}\right) x_{0}\right\|_{L_{2}} \leq c \omega_{2}\left(x_{0}, m^{-1}\right) \leq c m^{-1+\varepsilon}
$$

for any small $\varepsilon>0$. Using (7.7) instead of (5.5) we arrive at the following theorem.

THEOREM 7.1. Suppose that the exact solution of Abel's integral equation satisfies (7.1). Let $x_{\alpha, n, m}^{\delta}$ be the solution obtained from noisy data (1.5) when applying Tikhonov regularization (5.1), (5.2) for $s=0, P_{m, 0}=Q_{m}$.

Then in case of white noise (1.6) we obtain

$$
\left(\mathbf{E}\left\|x_{0}-x_{\alpha, n, m}^{\delta}\right\|_{L_{2}}^{2}\right)^{1 / 2} \leq c \delta^{1 / 2}
$$

which is achieved for $\alpha \asymp \delta^{1 / 2}, n \asymp \delta^{-1 / 2}$ and $m \asymp \delta^{-1 /(2-2 \varepsilon)}$ for any fixed $1>$ $\varepsilon>0$. 
We note that the information complexity $N^{r a n}\left(A, \mathcal{U}, X_{R}^{1}, \delta\right)$ can be achieved by the given histogram bins (1.5).

Remark 4. As already mentioned, the above result is not directly comparable with [29]. If we nevertheless would like to compare the respective assumptions and error estimates, then we would have to chose $\mu=2$ (i.e., $x_{0} \in \operatorname{Im}\left(\left(A^{*} A\right)^{2}\right)$ compared to $x_{0} \in \operatorname{Im}\left(A^{4}\right)$ in [29]) and the residual error corresponds to $\nu=-1 / 2$ in our case. This, together with the standard calibration $\delta=N^{-1 / 2}$, where $N$ is the sample size in [29], results in

$$
\left(\mathbf{E}\left\|A\left(x_{0}-x_{\alpha, n, m}^{\delta}\right)\right\|_{L_{2}}\right)^{1 / 2} \leq c \delta^{5 / 6}
$$

where $x_{\alpha, n, m}^{\delta}$ is constructed with appropriate choice of $m$ in a space of higher smoothness, such that (5.5) is satisfied. This rate formally agrees with the one in [29].

Moreover, under our more restrictive assumptions on the noise, the previous results yield that this order of accuracy is best possible.

\section{REFERENCES}

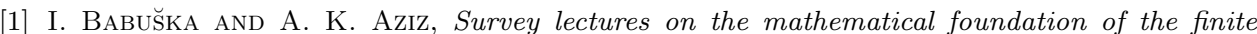
element method, in The Mathematical Foundation of the Finite Element Method with Applications to Partial Differential Equations, A. A. K., ed., Academic Press, New York, 1972, pp. 1-359.

[2] E. N. Belitser And B. Y. Levit, On minimax filtering over ellipsoids, Math. Methods Statist., 4 (1995), pp. 259-273.

[3] A. Böttcher, A. Dijksma, H. Langer, M. A. Dritschel, J. Rovnyak, And M. A. KAAshoek, Lectures on Operator Theory and Its Applications, AMS, Providence, RI, 1996. Lectures presented at the meeting held at the Fields Institute for Research in Mathematical Sciences, Waterloo, Ontario, September 1994, Edited by Peter Lancaster.

[4] G. Bruckner, S. Prössdorf, and G. Vainikko, Error bounds of discretization methods for boundary integral equations with noisy data, Appl. Anal., 63 (1996), pp. 25-37.

[5] L. Cavalier and A. B. Tsybakov, Sharp Adaptation for Inverse Problems with Random Noise, Preprint 559, Univ. Paris VI and Paris VII, 1999.

[6] P.-L. Chow, I. A. Ibragimov, And R. Z. Khasminskit, Statistical approach to some illposed problems for linear partial differential equations, Probab. Theory Related Fields, 113 (1999), pp. 421-441.

[7] W. Dahmen, A. Kunoth, And R. Schneider, Operator equations, multiscale concepts and complexity, in The Mathematics of Numerical Analysis, AMS, Providence, RI, 1996, pp. 225-261.

[8] V. DiCken And P. MaAss, Wavelet-Galerkin methods for ill-posed problems, J. Inverse Ill-Posed Prob., 4 (1996), pp. 203-221.

[9] D. Donoho, Nonlinear solution of linear inverse problems by wavelet-vaguelette decomposition, Appl. Comput. Harmon. Anal., 2 (1995), pp. 101-126.

[10] M. R. Dostanić, Asymptotic behavior of the singular values of fractional integral operators, J. Math. Anal. Appl., 175 (1993), pp. 380-391.

[11] H. W. Engl, M. Hanke, And A. Neubauer, Regularization of Inverse Problems, Kluwer Academic Publishers, Dordrecht, the Netherlands, 1996.

[12] H. W. Engl And A. Neubauer, Convergence rates for Tikhonov regularization in finitedimensional subspaces of Hilbert scales, Proc. Amer. Math. Soc., 102 (1988), pp. 587-592.

[13] G. Golubev and R. Z. Khasminski, Statistical Approach to Inverse Boundary Value Problems for Partial Differential Equations, Preprint 370, WIAS Berlin, 1997.

[14] M. Hegland, Variable Hilbert scale and their interpolation inequalities with applications to Tikhonov regularization, Appl. Anal., 59 (1995), pp. 207-223.

[15] I. M. Johnstone And B. W. Silverman, Discretization effects in statistical inverse problems, J. Complexity, 7 (1991), pp. 1-34.

[16] A. P. Korostelev and A. B. Tsybakov, Minimax Theory of Image Reconstruction, Lecture Notes in Statist. 82, Springer, New York, 1993.

[17] K. C. LI, Minimaxity of the method of regularization of stochastic processes, Ann. Statist., 10 (1982), pp. 937-942. 
[18] A. K. Louis, Inverse und schlecht gestellte Probleme, B. G. Teubner, Stuttgart, 1989.

[19] M. LukAs, Comparisons of parameter choice methods for regularization with discrete noisy data, Inverse Problems, 14 (1998), pp. 161-184.

[20] B. A. MAIR, Tikhonov regularization for finitely and infinitely smoothing operators, SIAM J. Math. Anal., 25 (1994), pp. 135-147.

[21] B. A. Mair And F. H. RuYmgaART, Statistical inverse estimation in Hilbert scales, SIAM J. Appl. Math., 56 (1996), pp. 1424-1444.

[22] P. MAthé, s-numbers in information-based complexity, J. Complexity, 6 (1990), pp. 41-66.

[23] V. A. Morozov, Methods for Solving Incorrectly Posed Problems, Springer-Verlag, New York, 1984.

[24] F. NATTERER, Regularisierung schlecht gestellter Probleme durch Projektionsverfahren, Numer. Math., 28 (1977), pp. 329-341.

[25] F. NATterer, Error bounds for Tikhonov regularization in Hilbert scales, Appl. Anal., 18 (1984), pp. 29-37.

[26] A. Neubauer, An a posteriori parameter choice for Tikhonov regularization in Hilbert scales leading to optimal convergence rates, SIAM J. Numer. Anal., 25 (1988), pp. 1313-1326.

[27] M. Nussbaum, Degrees of Ill-Posedness in Stochastic and Deterministic Noise Models, Report 101, Dagstuhl, 1994.

[28] M. Nussbaum and S. V. Pereverzev, The Degree of Ill-Posedness in Stochastic and Deterministic Noise Models, Preprint 509, WIAS Berlin, 1999.

[29] D. W. NychKa AND D. D. Cox, Convergence rates for regularized solutions of integral equations from discrete noisy data, Ann. Statist., 17 (1989), pp. 556-572.

[30] A. Pietsch, Operator Ideals, North-Holland, Amsterdam, 1980.

[31] S. G. Samko, A generalized Abel equation and fractional integration operators, Differencial' nye Uravnenija, 4 (1968), pp. 298-314.

[32] S. G. Samko, A. A. Kilbas, And O. I. Marichev, Integraly i proizvodnye drobnogo poryadka i nekotorye ikh prilozheniya, S. M. Nikol/skiü, ed., Nauka i Tekhnika, Minsk, 1987.

[33] J. SARAnen, A modified discrete spectral collocation method for first kind integral equations with logarithmic kernel, J. Integral Equations Appl., 5 (1993), pp. 547-567.

[34] E. ScHOCK, Ritz-regularization versus least-square-regularization. Solution methods for integral equations of the first kind, Z. Anal. Anwendungen, 4 (1985), pp. 277-284.

[35] P. Speckman, Spline smoothing and optimal rates of convergence in nonparametric regression models, Ann. Statist., 13 (1985), pp. 970-983.

[36] U. TAutenhahn, Error estimates for regularization methods in Hilbert scales, SIAM J. Numer. Anal., 33 (1996), pp. 2120-2130.

[37] A. N. Tikhonov and V. Y. Arsenin, Solutions of ill-posed problems, V. H. Winston \& Sons, Washington, D.C.: John Wiley \& Sons, New York, 1977. Translated from the Russian. Preface by translation editor Fritz John, Scripta Series in Mathematics.

[38] J. F. Traub, G. W. Wasilkowski, and H. Woźniakowski, Information-Based Complexity, Academic Press, Boston, MA, 1988.

[39] G. M. VaǏnikKo And U. A. Khyamarik, Projection methods and self-regularization in ill-posed problems, Izv. Vyssh. Uchebn. Zaved. Mat., 84 (1985), pp. 3-17.

[40] G. M. VAǏNIKKO AND A. Y. Veretennikov, Iteratsionnye protsedury $v$ nekorrektnykh zadachakh, Nauka, Moscow, 1986.

[41] G. WahbA, Practical approximate solutions to linear operator equations when the data are noisy, SIAM J. Numer. Anal., 14 (1977), pp. 651-667.

[42] A. G. Werschulz, The Computational Complexity of Differential and Integral Equations. An Information-Based Approach, The Clarendon Press, Oxford University Press, New York, 1991. 